\title{
Place of Doppler Ultrasound in the Characterization of Ovarian Cysts: About 35 Cases
}

\author{
Acko-Ohui Estelle Valérie ${ }^{1 *}$, Garba Idrissa ${ }^{2}$, Setchéou Alihonou ${ }^{2}$, Konan Anhum², Dédé Simon ${ }^{1}$ \\ ${ }^{1}$ Department of Radiodiagnosis and Medical Imaging, University Hospital (UH) of Treichville, Abidjan, Côte d'Ivoire \\ ${ }^{2}$ Department of Radiodiagnosis and Medical Imaging, University Hospital (UH) of Yopougon, Abidjan, Côte d'Ivoire \\ Email: *ohuiestelle@yahoo.fr, ackouv.67@gmail.com, toudouidriss@gmail.com, setcheoualiho@yahoo, \\ anhum_konan@yahoo.fr, simded@gmail.com
}

How to cite this paper: Estelle Valérie, A.-O., Idrissa, G., Alihonou, S., Anhum, K. and Simon, D. (2018) Place of Doppler Ultrasound in the Characterization of Ovarian Cysts: About 35 Cases. Open Journal of Radiology, 8, 167-174.

https://doi.org/10.4236/ojrad.2018.83019

Received: June 27, 2018

Accepted: August 26, 2018

Published: August 29, 2018

Copyright (C) 2018 by authors and Scientific Research Publishing Inc. This work is licensed under the Creative Commons Attribution International License (CC BY 4.0).

http://creativecommons.org/licenses/by/4.0/

\begin{abstract}
Pelvic ultrasound is the first-line examination for the diagnosis of ovarian cysts. When coupled with Doppler, it allows the diagnosis to be directed towards the benign or malignant nature of the cyst. These benign tumors are frequent in pubertal period. We report a descriptive study in 35 patients aged between 18 and 32 years of age who received a pelvic inflammatory etiology assessment. The purpose of this study was to show the place of pelvic ultrasound in the diagnosis of ovarian cysts.
\end{abstract}

\section{Keywords \\ Cyst, Ovary, Ultrasound, Doppler}

\section{Introduction}

An ovarian cyst is either benign in nature or malignant in nature. About $80 \%$ of ovarian tumors are cystic in nature and therefore benign [1]. According to the author Rubin [2], the benign nature of an ovarian cyst is difficult to assert from the outset before the ultrasound based solely on clinical examination. Most isolated adnexal masses are malignant or limited malignancy tumors according to Alcazar [3].

Pelvic ultrasound seems to be the gold standard for diagnosis of these cysts. The functional cyst is well appreciated by ultrasound [2]. The ovarian cyst is usually a benign tumor, often functional ( $15 \%$ to $45 \%$ of operated cysts), disappearing spontaneously within three months of its discovery [3]. According to the author Salem [4], the objectives of the practitioner who supports the patient are 
therefore to know the cyst to operate, how, and by whom? Clinical, endovaginal ultrasound and tumor markers are the most reliable means we have for characterizing an adnexal mass, but their positive predictive value remains low when it is a complex tumor, says Damarey [5]. The appearance of color Doppler since the early 1990s has improved diagnostic reliability in the evaluation of ovarian tumors [3].

Ultrasonography can be done by the endovaginal route and by the supra pubic route. Endovaginal ultrasound does not require a full bladder for its realization. On the other hand, the above-pubic approach requires bladder repletion but is nevertheless useful for an overview and for large tumors [3]. When coupled with Doppler, it allows the cyst to be oriented towards a benign or malignant character. The aim of this study is to show the interest of ultrasound in the characterization of ovarian cystic masses and to define what is acquired and what remains to be demonstrated in the field of ultrasound at a time when MRI is becoming the preferred technique for the pelvis in the face of pathology.

We report the case of a descriptive study of 35 patients whose age ranged from 18 to 32 years.

\section{Patients and Method}

This was a prospective descriptive study carried out over 6 months from January 2016 to June 2016 in an Imaging and Radiodiagnostic service of our city. It was based on the realization of pelvic ultrasound in all women with clinical information, pelvic pain or as part of a balance sheet of a cycle disorder. They were recruited from the outset in the study when their radiology bulletin was well informed. They were all informed about the progress of the study. Patients were monitored regularly. The study focused on the number, size, uni or plurilocular character, content and vascularization of the cyst. Pelvic ultrasound was performed by the pubic route in some patients and endovaginal in others. It was performed by the same operator and with the same device using a $3.5 \mathrm{MHZ}$ sector probe. The ultrasound examination was coupled to Doppler.

\section{Result}

Patient patients' ages ranged from 18 to 32 years, peaking at 25 years. During this study period, we recruited a total of 35 patients. They were all nulliparous. The menstrual cycle was regular in 28 women; ten of them had dysmenorrhoea.

The analysis of the wall, the content and the uni- or multi-cell aspect allowed us to classify the different cystic masses in these patients. Of these patients, 15 women had functional cysts (Figure 1); 8 had unilocular serous cystadenoma (Figure 2); 7 had an endometriotic cyst (Figure 3); 2 had a dermoid cyst (Figure 4); 3 others had thick-walled, thick-walled and heterogeneous-content cysts directed towards malignant masses (Figure 5(a) and Figure 5(b)). The latter benefited from a complementary pelvic MRI. The functional cyst is a fluid mass that disappears spontaneously within a few weeks or after two or three 


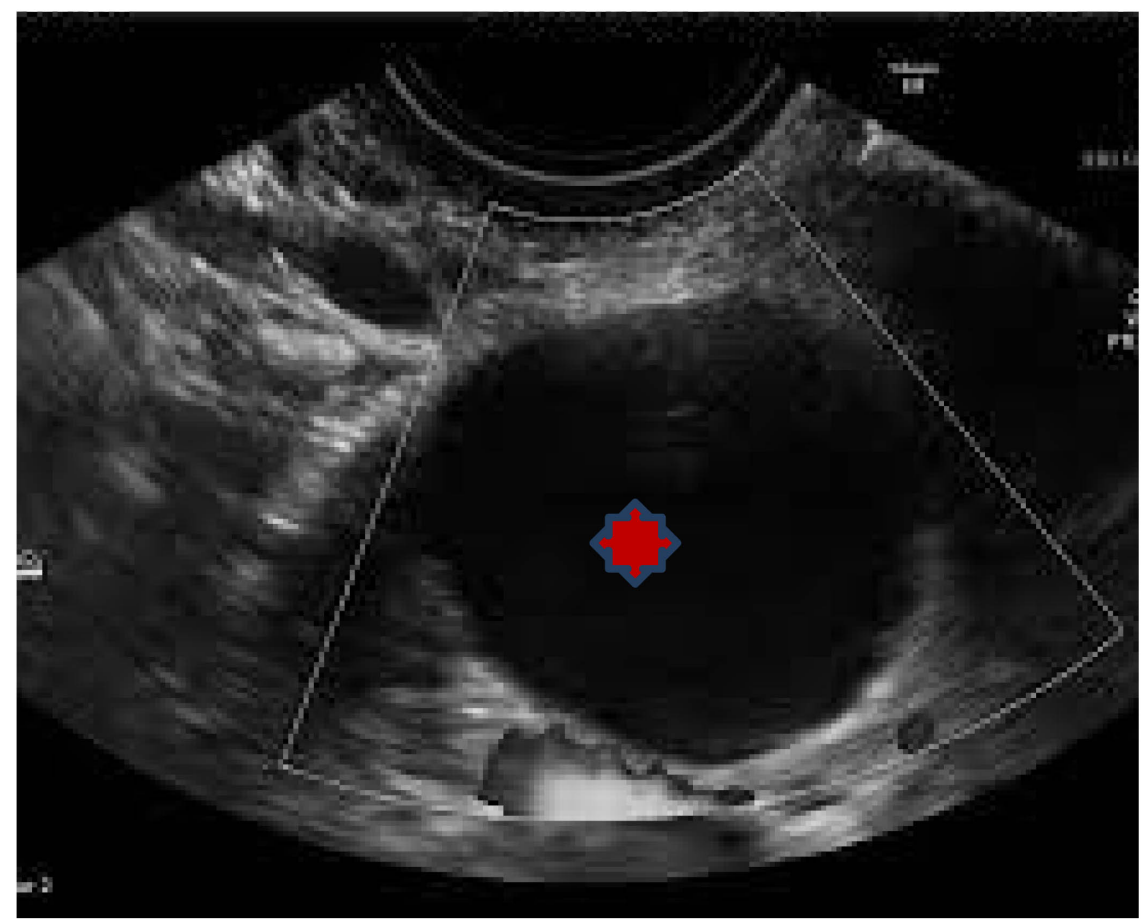

Figure 1. Pelvic ultrasound by the suprapubic route: functional cyst. Right ovarian mass of an anechoic structure without septum with posterior reinforcement with weak peripheral Doppler vascularization.

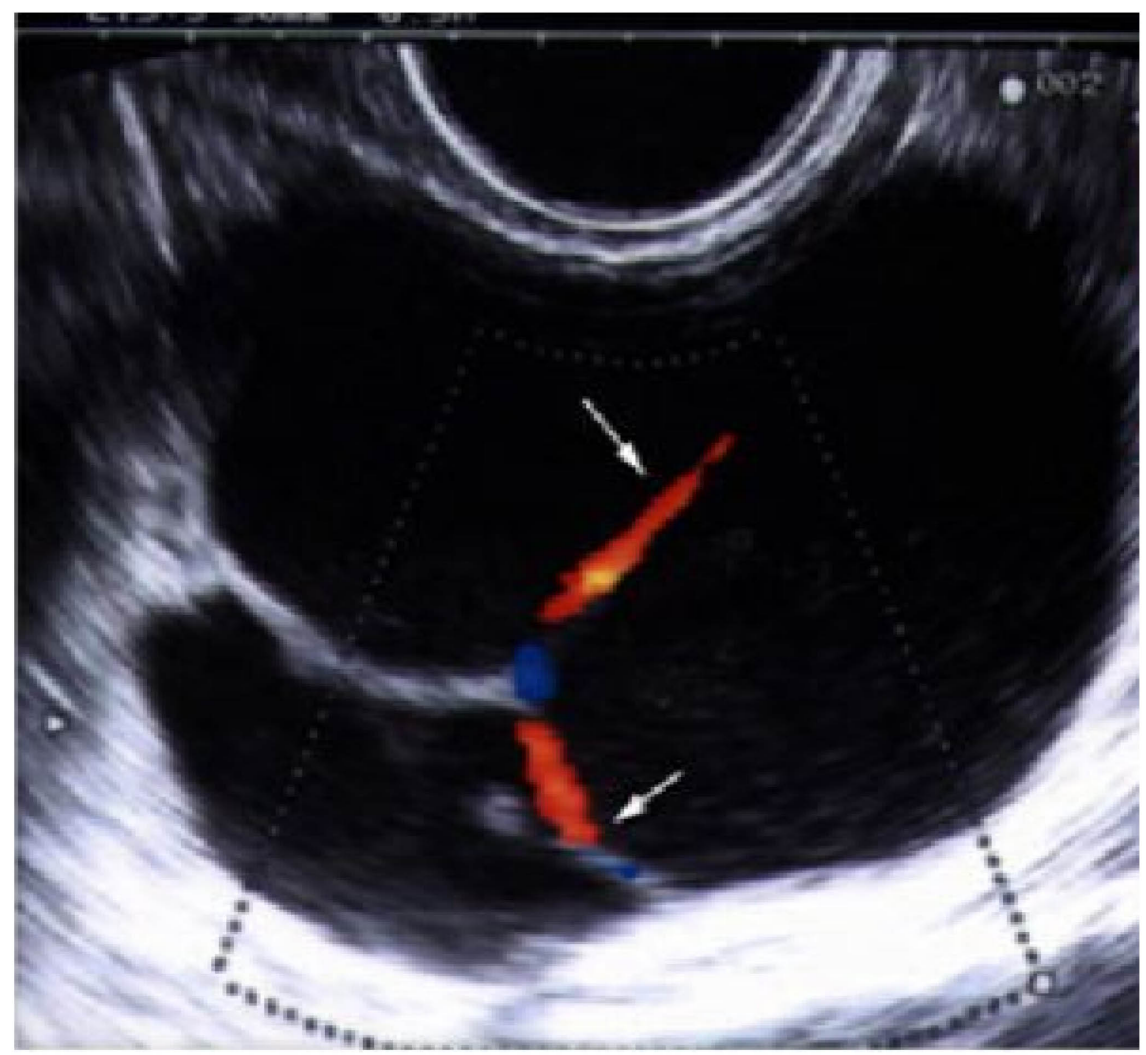

Figure 2. Endovaginal pelvic ultrasonography: unilocular serous cystadenoma. Anechoic left ovarian mass enclosing a thin vascularized Doppler wall with a regular wall. 


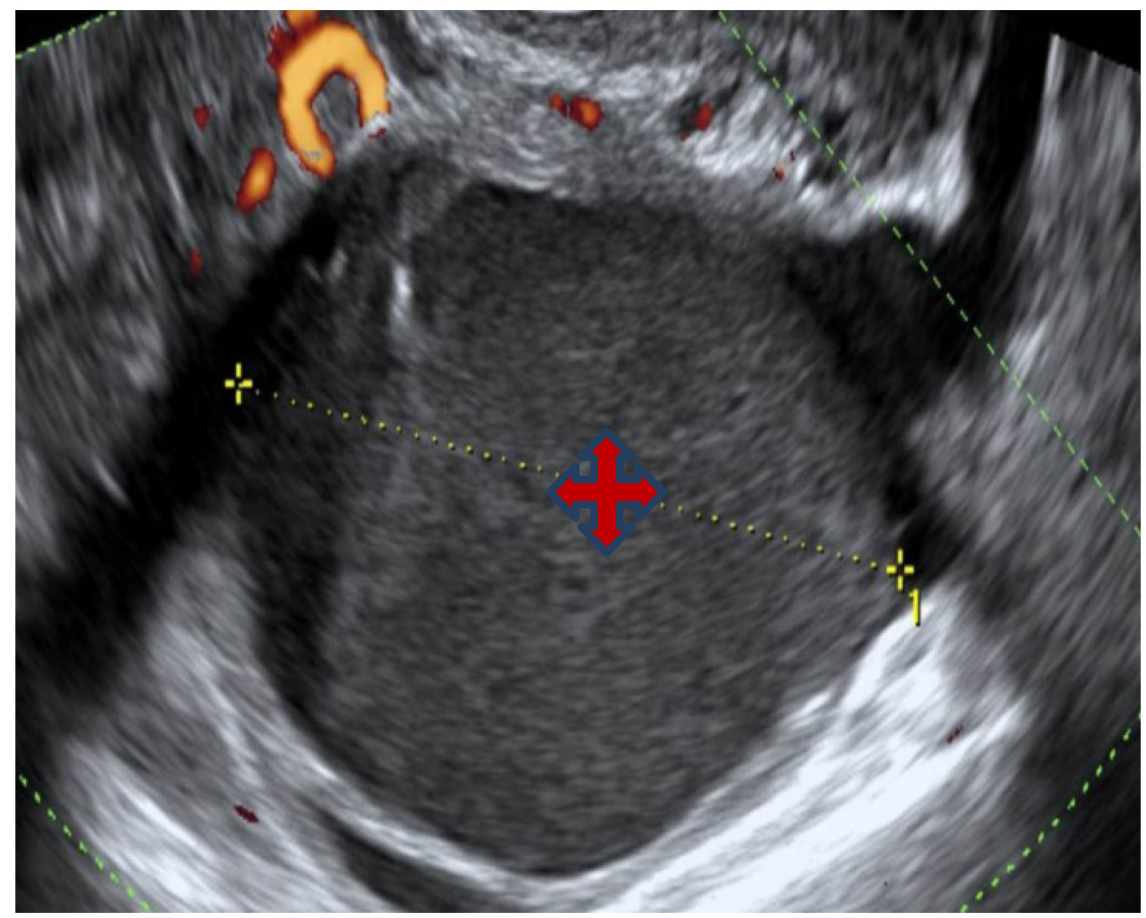

Figure 3. Endovaginal pelvic ultrasonography: endometriotic cyst. Right ovarian mass without septum containing fine echoes without intracystic vascularization.

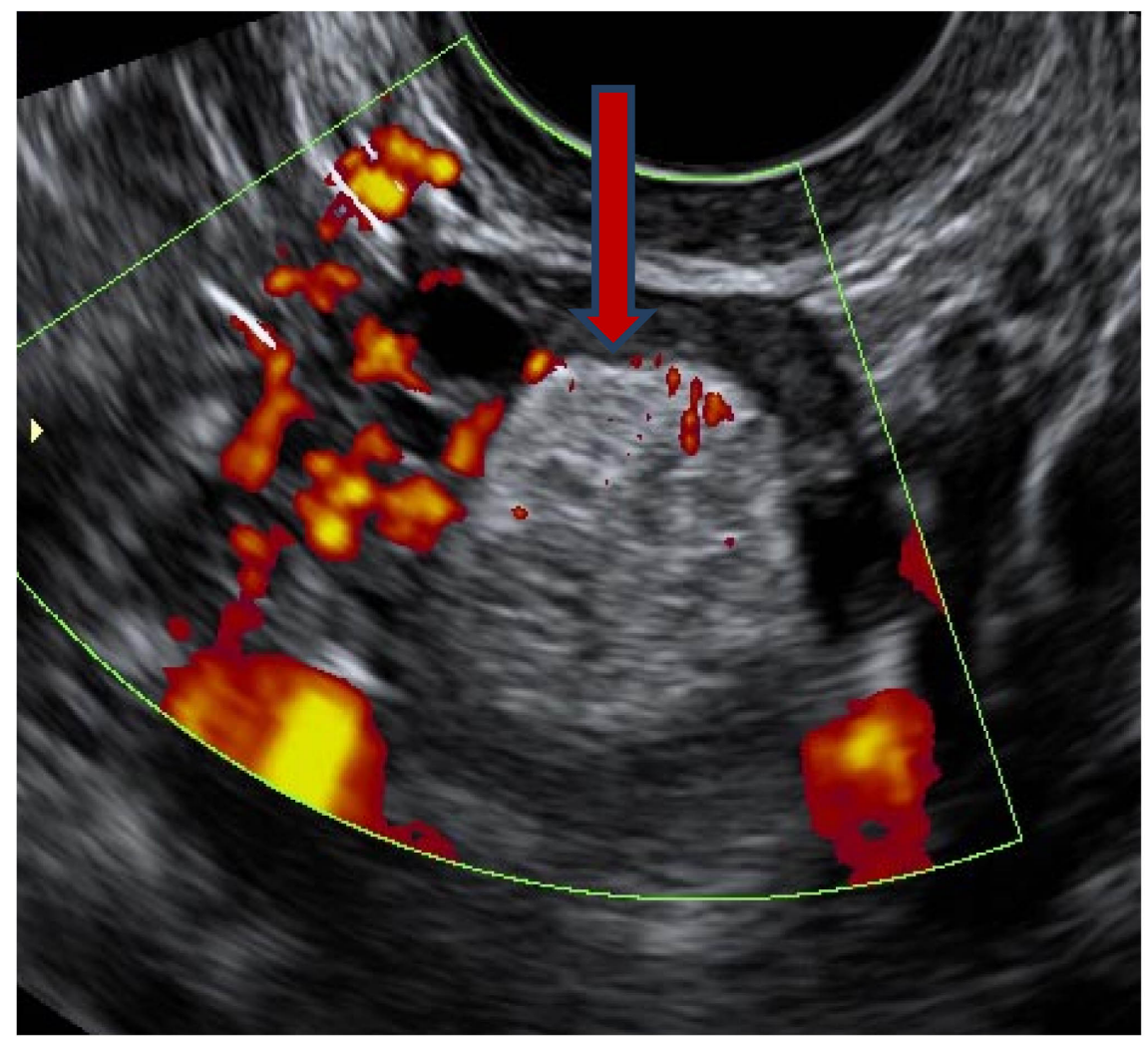

Figure 4. Endovaginal pelvic ultrasonography: dermoid cyst. Heterogeneous right ovarian mass with intracystic vascularization. 


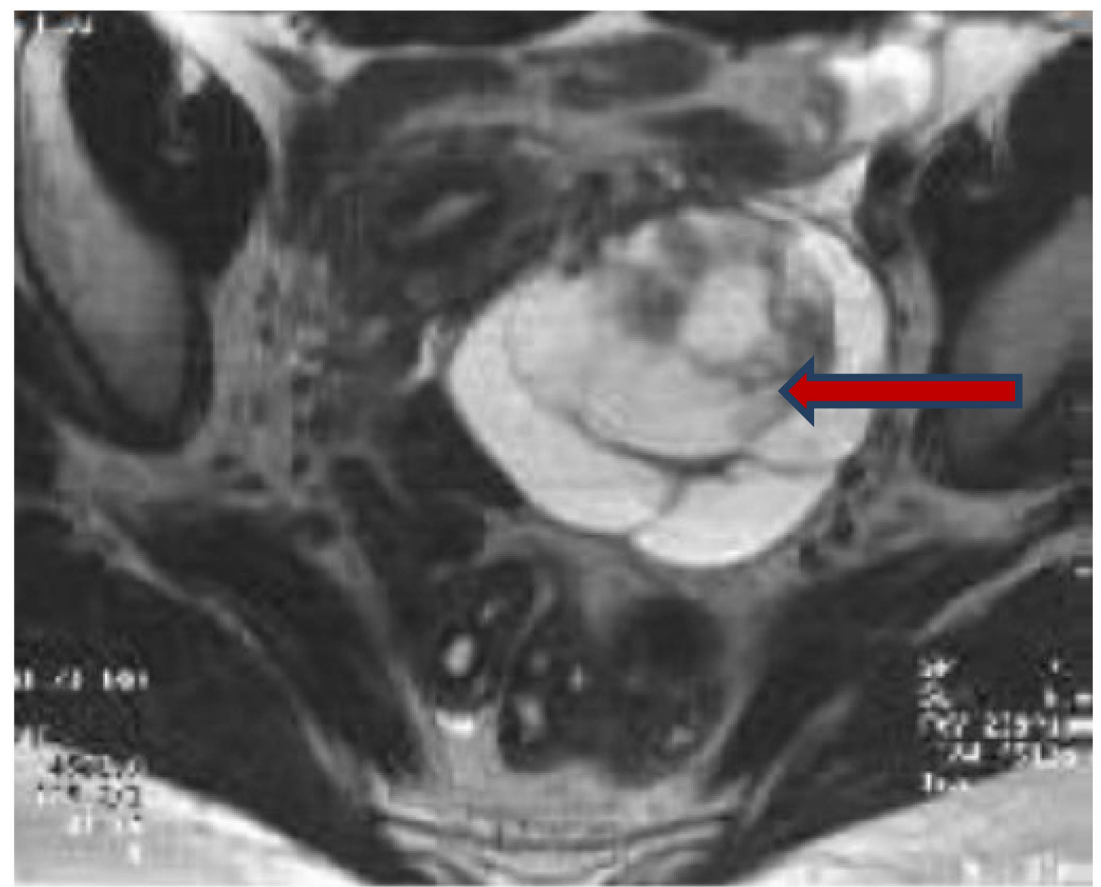

(a)

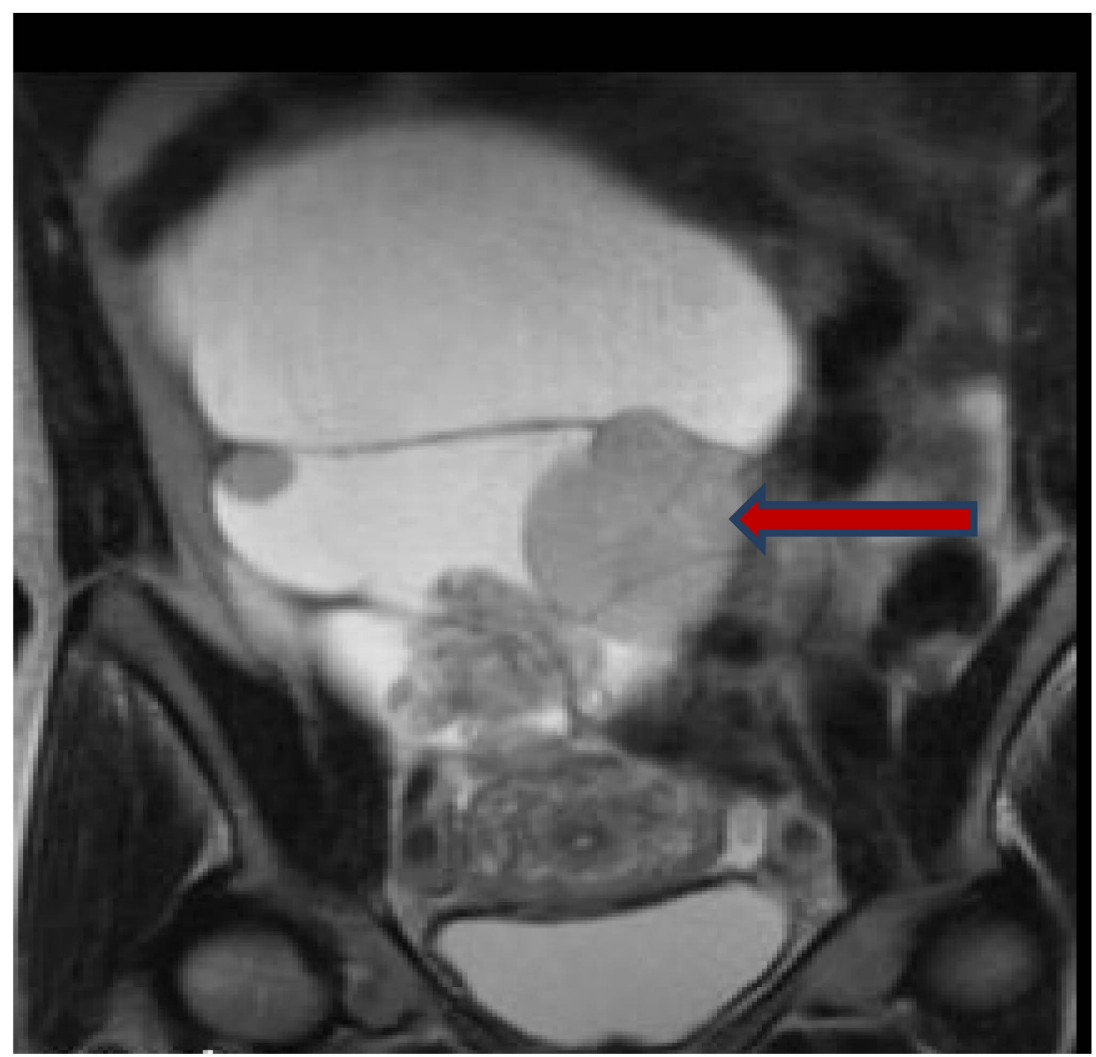

(b)

Figure 5. (a) pelvic MRI: axial section in EST2: serous cystanenocarcinoma. Thick parietal thick septate cyst with heterogeneous hypersignal vegetations; (b) Pelvic MRI: coronal cut in EST2: mucinous cystanenocarcinoma. Cyst containing multiple vegetations in hypersignal within which hyposignal partitions are found. 
menstrual cycles. The unilocular serous cystadenoma is a pure fluid tumor with clear contents, regular wall and distinguishing from the functional cyst by its persistent after several cycles. The dermoid cyst is a tumor of embryonic origin formed by several types of cells such as hair, hair, teeth, bones, cartilage giving this type of tumor a "monstrous" appearance. The endometriotic cyst corresponds to the ectopic localization of the endometrium within the ovary. It is characterized by the presence of fine echoes piqueted within it corresponding to intra-cystic bleeding at each menstrual cycle. She is a source of infertility. The cysts containing vegetations, thick and irregular wall, hyper vascularized Doppler are malignant cysts. Surgery of cysts coupled with the histological study of cysts which are of diagnostic value was not carried out in our study because of the limit of the finances and the refusal of some of our patients for the surgery.

\section{Discussion}

Pelvic ultrasound via the pubic approach coupled with Doppler is used to perform an accurate diagnosis of adnexal masses [3]. Some authors found a specificity of up to $85 \%$ to differentiate certain ovarian tumors [4]. Pelvic ultrasound can be used to analyze the cyst wall, the presence or absence of intracystic vegetation, the uni or multilocular character, the vascularization of the cyst by Doppler [5]. Doppler is directed towards the benign or malignant nature of this cystic mass by demonstrating a peripheral vascularization for the benign cyst and a central hypervascularisation for the malignant cyst [5] [6]. The diagnosis of dermoid cysts is also reliable, the hyperechoic component of which is concordant with histology in $96 \%$ of cases [6]. The finding of a bilateral tumor sometimes also directs the sonographer. The functional cyst is rarely bilateral. Conversely, the dermoid cyst and the serous cyst are bilateral in $20 \%$ of cases and the endometrioma in 50\% of cases [7]. We have objectified two cases of dermoid cyst. Dermoid cysts are characterized by well-defined and strongly echoic nodules of Rokitansky, thick white lines corresponding to hair and the presence of an acoustic shadow [6] [7]. When the cyst is unilocular fluid, the diagnostic orientation can be made either to the functional cyst or unilocular serous cystadenoma [7] [8]. In our study, functional cysts were identified in 15 patients. These cysts had a thin wall, an anechoic content without partition or vegetation, a weak peripheral vascularization and regressed at the end of the following cycle. Eight of them had a unilocular serous cystadenoma. These unilocular serous cystadenomas had the same characteristics as the functional cyst. It is their persistence after 3 months of control that made the diagnosis. The cyst may have a heterogeneous content especially in the context of endometriotic cysts where the contents are stitched with fine echoes [9]. Intra-cystic vascularization was poor. Seven of our patients had endometriotic cysts. Cysts containing vegetations, with thick and irregular walls, are suggestive of malignant masses and require other complementary examinations, notably MRI of the pelvis [10]. MRI of the pelvis characterizes these suspicious cystic masses which may be 
borderline serous cystadenoma, serous or mucinous cystadenocarcinoma [11] [12]. We isolated 3 cases of cystic mass suspected of malignancy. MRI performed in these patients led to the diagnosis of serous cystadenocarcinoma in two patients and mucinous cystadenocarcinoma in the other [12].

\section{Conclusion}

Pelvic ultrasonography coupled with Doppler is of paramount importance in the characterization of ovarian cystic masses. Doppler is mainly directed towards the benign or malignant aspect of the tumor. The pelvic ultrasound performed by a qualified sonographer and on a good device makes it possible to make an adequate diagnosis and consequently, directs the gynecologist in his care.

\section{Conflicts of Interest}

The authors declare no conflicts of interest regarding the publication of this paper.

\section{References}

[1] Takac, I. (1998) Receiver Operating Characteristic Curves of Transvaginaldoppler Blood Flow Measurements in Benign and Malignant Adnexal Tumors. Journal of Ultrasound in Medicine, 17, 637-642. https://doi.org/10.7863/jum.1998.17.10.637

[2] Rubin, J.M. (1994) Power Doppler US: A Potentially Useful Alternative to Mean Frequency-Based Color Doppler US. Radiology, 190, 853-856. https://doi.org/10.1148/radiology.190.3.8115639

[3] Alcazar, J.L. and Jurado, M. (1998) Using a Logistic Model to Predict Malignancy of Adnexal Masses Based on Menopausal Status, Ultrasound Morphology and Color Doppler Findings. Gynecologic Oncology, 69, 146-150. https://doi.org/10.1006/gyno.1998.4995

[4] Salem, A., Fakhfekh, K., Rajhi, H., Ben Temime, R., Koubaa, A. and Mnif, N. (2009) Imagerie en Pratique Clinique. La Tunisie Medicale, 87, 334-339.

[5] Damarey, B., Farine, M.O., Vinatier, D., Collinet, P., Lucot, J.P., Kerdraon, O. and Poncelet, E. (2010) Tératomes ovariens matures et immatures: Caractéristiques en échographie, TDM et IRM. Journal de Radiologie, 91, 27-36.

https://doi.org/10.1016/S0221-0363(10)70003-2

[6] Douay-Hauser, N., Koskas, M., Walker, F., Luton, D. and Yazbeck, C. (2011) Diagnosis and Management of an Immature Teratoma during Ovarian Stimulation: A Case Report. Journal of Medical Case Reports, 5, 540.

[7] Valentin, L. (1999) Prospective cross Validation of Doppler Ultrasound Examination and Gray-Scale Ultrasound Imaging for Discrimination of Benign and Malignant Pelvic Masses. Ultrasound in Obstetrics \& Gynecology, 14, 273-283. https://doi.org/10.1046/j.1469-0705.1999.14040273.x

[8] Rantomalalaa, H.Y.H., Ravelosona, J.R., Rakotoarisoaa, B., Rabesataa, J.O. and Tovoneb, X.G. (2003) Une fistulisation vésicale d'un kyste dermoïde de l'ovaire. Annales d Urologie, 37, 102-104. https://doi.org/10.1016/S0003-4401(03)00035-4

[9] Aslam, N., Banerfee, S., Carr, J., Savvas, M., Hooper, R. and Jurkovic, D. (2000) Prospective Evaluation of Logistic Regression Models for the Diagnosis of Ovarian Cancer. Obstetrics \& Gynecology, 96, 75-80. 
[10] Mol, B., Boll, D., De Kanter, M., Heintz, P., Sijmons, E., Oei, S., Bal, H. and Brölmann, H. (2001) Distinguishing the Benign and the Malignant Adnexal Mass: An External Validation of Prognostic Models. Gynecologic Oncology, 80, 162-167. https://doi.org/10.1006/gyno.2000.6052

[11] El Mansouri, A. and Aderdour, M. (1992) Tumeurs de l'ovaire et grossesse (A propos de 30 cas). Médecine du Maghreb, 33, 29-34.

[12] Kinkel, K., Hricak, H., Lu, Y., Tsuda, K. and Filly, R.A. (2000) US Characterization of Ovarian Masses: A Meta-Analysis. Radiology, 217, 803-811.

https://doi.org/10.1148/radiology.217.3.r00dc20803 